\title{
Conditioning the GSR and digital vasomotor response to words under a short and a long CS-UCS interval
}

\author{
IRVING MALTZMAN and DAVID C. RASKIN \\ University of California, Los Angeles, Los Angeles, California 90024
}

\begin{abstract}
Sixty college students served in an experiment in which the GSR and digital vasomotor response were conditioned to words. Half the students were conditioned with a .5 -sec CSUCS interval and half with a 10 -sec CS-UCS interval. There were nine reinforced trials where the CS was followed by a 1-sec 110-dB noise and five interspersed test trials where the UCS was omitted. A significant CS-UCS Interval by Words interaction was obtained for the GSR and for the digital vasomotor response. Differential conditioning of the GSR was better under the .5- than under the 10-sec interval. In contrast, differential conditioning of the vasomotor response was better under the 10- than under the .5-sec interval. Different optimal CS-UCS intervals for the two kinds of differential conditioned responses appeared to have their effect as a consequence of performance differences rather than a difference in learning.
\end{abstract}

Jones (1962) has taken the position that the strength of classical aversive conditioning not only depends upon the CS-UCS interval, but is also a function of the latency of the response conditioned. Responses with different latencies will have different optimal intervals for conditioning. Jones (1962) tested her theory in a simple aversive conditioning situation by simultaneously conditioning finger movement and the GSR. Her findings confirmed the prediction that the longer latency GSR has an optimal CS-UCS interval which is longer than that for the shorter latency finger withdrawal response. A study by Champion (1962) employing the GSR and eyeblink response added further support to Jones' hypothesis.

A possible criticism of these studies is that in both cases the short-latency responses were skeletal and the long-latency response autonomic in nature. Optimal intervals may therefore be peculiar to these different response systems. Two extensive experiments (Maltzman, Gould, Barnett, Raskin, \& Wolff, 1977) compared differential conditioning of two autonomic responses to words under an extended range of intervals. Conditioning of the GSR and digital vaso-

This study was made possible in part by funds provided by United States Public Health Service Grant MH 04684 and from the Carnegie Corporation of New York. For the analyses of the results of the experiment reported here, computing assistance was obtained from the Health Science Computing Facility, University of California, Los Angeles, sponsored by National Institutes of Health Grant FR-3. Portions of the results obtained in this experiment have been presented elsewhere (Maltzman, 1968). David C. Raskin is now at the University of Utah. Requests for reprints should be addressed to Irving Maltzman, Department of Psychology, University of California, Los Angeles, California 90024. motor response (VMR) activity to words was accomplished in different groups under CS-UCS intervals of $.5,5.5,10.5,15.5$, and $20.5 \mathrm{sec}$. Extinction showed generally superior GSR conditioning under the short intervals as compared to the long intervals. All intervals showed reliable differential conditioning. Differential conditioning of the digital VMR under the various intervals did not differ significantly. Essentially comparable results were obtained in the two experiments, one with a noxious UCS and the other using an innocuous tone in a forewarned reaction time situation.

An analysis by Badia and Defran (1970) of effects obtainable during conditioning with short and long CS-UCS intervals has questioned the interpretation of all such studies. They essentially deny associative status to responses obtained under UCS omission trials when a short CS-UCS interval is employed. Omitting the UCS constitutes a change in the CSUCS stimulus complex and may induce an orienting reflex (OR) reflected as an augmented GSR to the omission of the UCS. This stimulus-sequence-changeelicited OR is confounded with the CR when the latency of the CR exceeds the CS-UCS interval. When longer CS-UCS intervals are employed, the response to UCS omission can be differentiated from the CR induced by the CS. While this stimulussequence-change argument seems logically reasonable, Furedy and Poulos (1977) conclude that the argument is an empirical red herring. Evidence from a variety of experiments fails to support the hypothesis that a stimulus sequence change of the kind found with omission of the UCS on a test trial evokes a nonassociative OR. In addition, Maltzman 
et al. (1977) reported that the GSRs to the control words preceding and following the CS word during semantic conditioning varied significantly with the CS-UCS interval in the same manner as the GSRCRs evoked during extinction. Responses to the control words under the .5-sec interval were significantly greater than the responses under the longer intervals when a noxious UCS was employed. Significant differences in the trends to the control words as a function of CS-UCS intervals during conditioning cannot be contaminated by possible ORs to stimulus changes during extinction as a consequence of UCS omission. Evidence for the superiority of the $.5-\mathrm{sec}$ interval in differential GSR conditioning was therefore obtained in the absence of possible contamination by stimulus-sequence-change-induced ORs. A similar effect was not obtained in the VMRs induced by control words in that same experiment. Occurrence of a significant difference in the GSRs induced by control words under different CS-UCS intervals and Furedy and Poulos' (1977) review of the relevant literature suggest that further examination of the effects of long and short CS-UCS intervals on the conditioning of autonomic responses is in order.

The purpose of the present experiment was to assess conditioning of two autonomic responses that differ somewhat in their onset latency. The GSR with a latency of approximately $1.5-2.5 \mathrm{sec}$ and digital blood volume change with a latency of approximately 1.5-4 sec were compared using the method of test trials during conditioning rather than resistance to extinction.

\section{METHOD}

\section{Subjects}

There were 60 University of California, Los Angeles introductory psychology students who participated in the experiment for partial fulfillment of a course requirement. Thirty students were conditioned in the short interval and 30 in the long CSUCS interval, with equal numbers of men and women under each condition.

\section{Procedure}

The experimental arrangement has been previously described (Maltzman et al., 1977). Words and the UCS, a 1-sec 110-dB (A) noise calibrated at the earphones, were prerecorded on magnetic tape, played back on a stereophonic tape deck, amplified, and presented via stereophonic headphones.

The GSR electrodes were two $20-\mathrm{mm}$ disk electrodes fitted in 5-mm-deep plastic cups, which were filled with electrode paste made from agar and $1 \%$ zinc sulfate solution and attached to the palms by plastic pressure clips. The GSR was recorded using a 40- $\mu \mathrm{A}$ Wheatstone bridge and one channel of a Sanborn 320 dual dc amplifier-recorder. The VMR transducer was a Starling Corp. photoplethysmograph. The light-photocell face of the transducer was in contact with the skin on the ventral side of the left index finger. Its signal was recorded on the second channel of the dc amplifier-recorder.

Prior to the start of conditioning, a habituation series of 20 neutral words were presented. These words were high-frequency, common words not associated with the CS word "light." Con- ditioning following habituation without interruption and consisted of 14 presentations of the CS word interspersed among neutral filler words. Half the students were conditioned with a CS-UCS interval of approximately $.5 \mathrm{sec}$ between the onset of the word and onset of the UCS, while half were conditioned under a 10 -sec CS-UCS interval. The UCS was omitted on five of the trials, 2, 5, 7, 11, and 13. From two to eight filler words intervened between each conditioning trial, reinforced and test. Time between successive words and the UCS and following word varied between 8 and $12 \mathrm{sec}$.

\section{RESULTS AND DISCUSSION}

The GSR was scored as the largest resistance change occurring within the $.5-8$-sec interval following the CS word "light" and the control words immediately preceding $\left(\mathrm{C}_{1}\right)$ and following $\left(\mathrm{C}_{2}\right)$ each reinforced and test trial. Blood volume changes were recorded in millimeters of pen deflection corrected for recorder sensitivity. These VMR changes were scored as the largest change in the lowest point of the systolic pulse in the .5-8-sec interval following the CS word and the control words. Prior to analysis, the GSR measure was subjected to a log conductance change transformation and the VMR was subjected to a square root difference transformation. The .05 level of significance for a two-tailed test was adopted in all statistical analyses.

The VMR data of four students were discarded, two because they contained extensive movement artifacts and two, randomly, to equate the groups.

Results obtained from the five conditioning test trials are shown in Figure 1. Mean magnitude of the GSRs evoked by the CS word and the preceding $\left(C_{1}\right)$ and following $\left(C_{2}\right)$ control words under the .5and 10 -sec CS-UCS intervals are shown in the upper two panels. Mean magnitude of the VMRs evoked by the CS word and the preceding $\left(C_{1}\right)$ and following
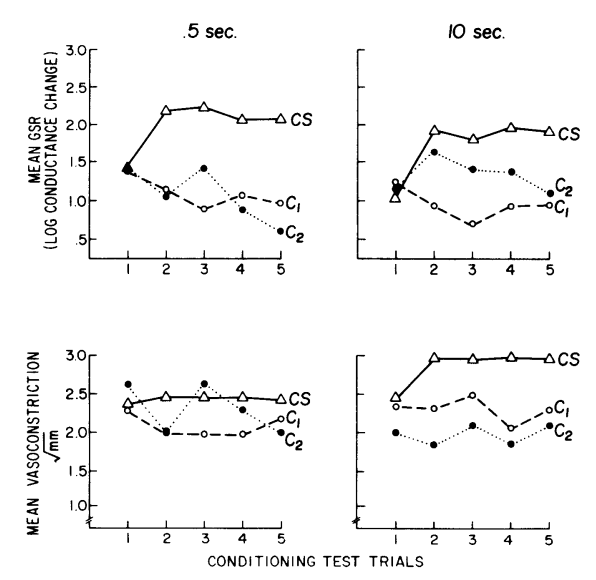

Figure 1. Mean magnitude of the galvanic skin response (GSR) and the digital vasomotor response (VMR) to the conditioned stimulus $(C S)$ and preceding $\left(C_{1}\right)$ and following $\left(C_{2}\right)$ control words as a consequence of conditioning under a .5 -sec or a 10 -sec CS-UCS interval. 
$\left(C_{2}\right)$ control words are shown in the two lower panels.

Analysis of variance of the GSR results indicated that reliable overall differential conditioning occurred, as reflected by a significant main effect of Words $[F(2,112)=73.31]$. More to the point, there was a significant interaction between CS-UCS intervals and Words $\left[\mathrm{F}(2,112)=9.47, \mathrm{MS}_{\mathrm{W}}=.765\right]$. Examination of Figure 1 suggests that reliably greater differential conditioning occurred under the .5 -sec than under the 10-sec CS-UCS interval. The difference, however, is largely due to the heightened responsivity induced by the $\mathrm{C}_{2}$ control word in the long CS-UCS interval. Although the GSRs induced by the CS word in the short interval were somewhat greater in magnitude than the CRs induced by the CS in the long interval, the difference is not significant. Results obtained when extinction tests were used revealed a significantly larger CR magnitude under the .5-sec interval than under a 10.5-sec CS-UCS interval (Maltzman et al., 1977). Examination of Figure 1 suggests that omission of the UCS under a long interval results in the evocation of the GSR-OR, or disparity response, when a $C_{2}$ word occurs in place of the anticipated UCS. Heightened responsivity to the $\mathrm{C}_{2}$ does not occur under the short .5 -sec interval because the participants can readily discriminate the short CS-UCS interval with omission of the UCS from the long interval lapsing prior to the occurrence of $\mathrm{C}_{2}$.

Analyses of variance of the VMR results indicated that reliable overall differential conditioning of the VMR occurred $[F(2,104)=8.30]$. A significant interaction between CS-UCS intervals and Words was also obtained for the $\operatorname{VMR}\left[\mathrm{F}(2,104)=5.89, \mathrm{MS}_{\mathrm{W}}\right.$ $=2.274]$. Examination of the lower panels in Figure 1 indicates an absence of differential conditioning under the .5 -sec interval but good differential conditioning under the 10 -sec interval. The magnitude difference between CRs, however, is not significant. In contrast to the GSR results under the long CSUCS interval, the VMR did not show heightened responsivity to the $C_{2}$ response. Absence of a heightened VMR to $C_{2}$ during extinction has also been found (Maltzman et al., 1977). This lack of correspondence between GSR and VMR results is in accord with the repeated reports that, while digital VMR and GSR are taken as indices of the OR, they nevertheless do not display the same characteristics under all conditions. Vasomotor activity is less sensitive to the kinds of stimulus change and novelty that ordinarily evoke a GSR-OR (Barry, 1977; Furedy, 1968; Maltzman et al., 1977; Ray, Piroch, \& Kimmel, 1977).

Jones' (1962) thesis that the optimal interval for conditioning will vary with the latency characteristics of the response finds some support in the present experiment. However, it is important to note that the differences in conditioning obtained in the present experiment largely are a consequence of the nature of the responses to the control words obtained under the two CS-UCS intervals for the GSR and VMR.

Superiority of the short interval for differential conditioning of the GSR was not the result of an augmented CR; it was a consequence of the lack of a large GSR to $\mathrm{C}_{2}$ following the UCS in contrast to the large response induced by $\mathrm{C}_{2}$ in the long CSUCS interval. Under the .5-sec CS-UCS interval, the student can readily discriminate the CS-UCS interval from the interval between the UCS and the $\mathrm{C}_{2}$ word because of the briefness of the delay between the CS and the UCS in contrast to the relatively long interval between the UCS and the following $\mathrm{C}_{2}$ word. It is this responsivity to the $C_{2}$ on a test trial or during extinction (Maltzman et al., 1977) that makes for poorer differential conditioning of the GSR in the long interval as compared with the short interval. Differential conditioning of the VMR was better in the long than in the short interval for a different reason. Manifest differential conditioning did not occur in the short interval, whereas the long interval yielded good differential responding. Differentiation did not suffer from a large response to $C_{2}$ in the long CS-UCS interval.

Results obtained in this study suggest that the optimal CS-UCS interval may differ for different responses, but there does not seem to be some underlying general principle or common physiological mechanism at work. Different intervals, and different experimental procedures, appear to yield superior differential conditioning under one or another interval for a variety of different reasons. Variations in the efficacy of different intervals seem to occur because of different kinds of effects on performance rather than different degrees of learning. At least some of these performance differences are induced by the nature of the differential as contrasted with simple conditioning. For example, the presence or absence of a large response to a control word following omission of the UCS in contrast to the response following the presentation of a UCS can be a major determiner of the degree of differential conditioning under different CS-UCS intervals, whereas it would not be a factor in simple conditioning.

A further qualification must be placed upon the obtained results. As is always the case, experimental findings are a function of, and limited by, the instrumentation employed, methods of measurement, scoring conventions, statistical model used, etc. It is difficult to employ corresponding measures for different autonomic responses. It is difficult even to employ equivalent measures for the same response when different scoring or CS-UCS intervals are used (Badia \& Defran, 1970). Since there is a much shorter 
history of research on the VMR than on the GSR, its characteristics under a variety of experimental conditions and scoring conventions are less well known than those of the GSR. Despite a variety of differences in methodology, the GSR tends to give similar results under similar experimental conditions. It is a robust index. Much less information is available concerning the characteristics of the VMR employing different methods of measurement and scoring conventions under a variety of experimental conditions. Since the present scoring convention differs from one often used (Furedy, 1968), it is possible that different results for the VMR would be obtained if a different scoring convention were employed. We do not believe that such would be the case, but it is an empirical issue and must await further systematic research.

\section{REFERENCES}

Badia, P., \& Defran, R. H. Orienting responses and GSR conditioning: A dilemma. Psychological Review, 1970, 77, 171-181.

BARRY R. J. Failure to find evidence of the unitary OR concept with indifferent low-intensity auditory stimuli. Physiological Psychology, 1977, 5, 89-96.
Champion, R. A. Stimulus-response contiguity in classical aversive conditioning. Journal of Experimental Psychology, 1962, 64, 35-39.

FUREDY, J. J. Human orienting reaction as a function of electrodermal versus plethysmographic response modes and single versus alternating stimulus series. Journal of Experimental Psychology, 1968, 77, 70-80.

Furedy, J. J., \& Poulos, C. X. Short-interval classical SCR conditioning and the stimulus-sequence-change-elicited OR: The case of the empirical red herring: Psychophysiology, 1977, 14, 351-359.

JoNES, J. E. Contiguity and reinforcement in relation to CS-UCS intervals in classical aversive conditioning. Psychological Review, 1962, 69, 176-186.

Maltzman, I. Theoretical conceptions of semantic conditioning and generalization. In T. R. Dixon \& D. L. Horton (Eds.), Verbal behavior and general behavior theory. Englewood Cliffs, N.J: Prentice-Hall, 1968.

Maltzman, I., Gould, J., Barnett, O. J., Raskin, D. C., \& WolfF, C. Classically conditioning components of the orienting reflex to words using an innocuous and a noxious UCS under different CS-UCS intervals. Journal of Experimental Psychology: General, 1977, 106, 185-212.

Ray, R. L., Piroch, J. R., \& Kimmel, H. D. The effect of task and stimulus variability on habituation of electrodermal and vasomotor reactions. Physiological Psychology, 1977, 5, 189-196.

(Received for publication February 1, 1978; revision accepted January $9,1979$. 